\title{
RepPop: a database for repetitive elements in Populus trichocarpa Fengfeng Zhou ${ }^{1,2}$ and Ying $\mathrm{Xu}^{* 1,2}$
}

\author{
Address: ${ }^{1}$ Computational Systems Biology Laboratory, Department of Biochemistry and Molecular Biology, and Institute of Bioinformatics, \\ University of Georgia, Athens, GA 30602, USA and 2BioEnergy Science Center, Tennessee, USA \\ Email: Fengfeng Zhou - ffzhou@csbl.bmb.uga.edu; Ying Xu* - xyn@bmb.uga.edu \\ * Corresponding author
}

Published: 9 January 2009

BMC Genomics 2009, I0:14 doi:10.1186/147|-2164-10-14

This article is available from: http://www.biomedcentral.com//47/-2/64/10/14

(c) 2009 Zhou and Xu; licensee BioMed Central Ltd.

This is an Open Access article distributed under the terms of the Creative Commons Attribution License (http://creativecommons.org/licenses/by/2.0), which permits unrestricted use, distribution, and reproduction in any medium, provided the original work is properly cited.

\begin{abstract}
Background: Populus trichocarpa is the first tree genome to be completed, and its whole genome is currently being assembled. No functional annotation about the repetitive elements in the Populus trichocarpa genome is currently available.

Results: We predicted 9,623 repetitive elements in the Populus trichocarpa genome, and assigned functions to 3,075 of them (31.95\%). The 9,623 repetitive elements cover $\sim 40 \%$ of the current (partially) assembled genome. Among the 9,623 repetitive elements, 668 have copies only in the contigs that have not been assigned to one of the 19 chromosome while the rest all have copies in the partially assembled chromosomes.

Conclusion: All the predicted data are organized into an easy-to-use web-browsable database, RepPop. Various search capabilities are provided against the RepPop database. A Wiki system has been set up to facilitate functional annotation and curation of the repetitive elements by a community rather than just the database developer. The database RepPop will facilitate the assembling and functional characterization of the Populus trichocarpa genome.
\end{abstract}

\section{Background}

The Poplar was selected to be the first tree genome to be sequenced, mainly because of its extraordinarily rapid growth rate and its relatively compact genome size (450500 Mbps [1,2]). Biofuels are produced mainly through two sources, i.e. crops high in sugar or cellulose, e.g. sugar canes [3] and plants [4], and plants high in vegetable oils like soybean[5]. The Populus trichocarpa genome's rapid growth coupled with the high content of lignocelluloses has made it one of the model systems for the new generation of biofuels [4]. The current assembly of the Poplar genome was released in June 2004, and its total length is 485 Mbps. The assembled 19 chromosomes with 7.66\% gaps count for $63.41 \%$ of the whole genome. Further efforts are still needed to close the gaps in the sequenced chromosomes.

Repetitive elements represent a significant fraction of eukaryotic genomes and they could occupy as high as $80 \%$ of some land-plant genomes like wheat [6] and as low as $10-35 \%$ for Arabidopsis thaliana [7] and rice [8]. There are three main classes of repetitive elements, namely, local repeats (tandem and satellite repeats) [9], interspersed repeats (transposons) and segmental duplications (duplicated genomic segments). Among them, transposable elements are the most extensively studied repetitive elements, and they can be classified as retrotransposons or DNA transposons based on whether they 
are transposed through the RNA or DNA intermediates [10]. Both interspersed repeats [11-16] and other duplicated elements [17] may induce homologous recombinations and insertions/deletions in the host genome, which may introduce great difficulties to the correct assembly of the repetitive regions in the host genome.

Typically repetitive elements have been identified in a genome using two approaches: (1) identification of homologous sequences to known repetitive elements [18], and (2) identification of repeats based on self-comparison a given genome and clustering them into families [19-21]. The first approach requires manually curated repetitive elements, which may not be feasible for newly sequenced genomes, though it can identify the precise boundaries of repetitive elements, even for the embedded partial copies. The second approach identifies repetitive elements in a de novo fashion, though it may require additional manual curations for the boundaries of the predicted elements.

\section{Construction and content Data resources}

The current assembly of the Populus trichocarpa genome was released in June 2004 as version 1.1, which consists of 22,012 nucleotide sequences, covering large pieces of the 19 chromosomes and some unassembled short contigs, and the total length is $485,510,911$ bps. This data was downloaded from the web site of Populus trichocarpa genome sequencing project [22].

We downloaded four of the most comprehensive databases of repetitive elements in eukaryotes, RepBase [23] version 12.05 (release of July 13, 2007), TREP [24] version 10 (release of July 2008), RetrOryza [25] and AtRepBase [26], for homology search. We also downloaded the databases RDP [27] and Rfam [28], and RNA genes in the rice RAP-DB database [29]. The NCBI database NT [30] containing all the non-redundant protein sequences was also downloaded for homology search.

\section{Identification of repetitive elements}

Due to the very large computer memory requirement by many repeat identification programs [19-21], we implemented our RepPop database and associated tools on a 64bit Linux operating system with 32 GB memory. The repetitive elements with at least 2 copies in the Poplar genome were identified using RepeatScout [19]. We then removed any repetitive elements predicted to be low complexity regions using program NSEG [31] and tandem repeats using program TRF [32]. All the programs were run using the default parameters.

Totally 9,623 repetitive elements were identified, covering $194.00 \mathrm{Mbps}(\sim 40 \%)$ of the Poplar genome. The distribu- tions of copy numbers and lengths of these repetitive elements are given in Figure 1. Most of the repetitive elements are short and of low copy numbers.

\section{Annotation procedure}

We first identified the homologous regions of the 9,623 repetitive elements in the databases RepBase [23], TREP [24], RetrOryza [25] and AtRepBase [26] using the NCBI Blast [30] with E-value cutoff e-5. One region might match two homologous elements in the database. We then removed the redundant annotations by keeping only the region with the lowest E-value for the overlapping regions. A total of 226 homologous regions were identified.

We then predicted 30 tRNA genes using the program tRNAscan-SE with default parameters [33]. 8 and 40 homologous regions to the RNA genes in databases $R D P$ [27] and RAP-DB [29] were identified using the NCBI Blast [30] with E-value cutoff e-5 after removing the redundancy like above. No homologous regions were identified based on the RNA profiles of Rfam [28] using the program infernal [19] with default parameters.

2,720 homologous regions to sequences in the database NT [30] were identified using NCBI Blast [30] with E-value cutoff e-5, and annotated as having the functions of the best matched homologous proteins.

\section{Utility and discussion}

We organized the 9,623 predicted repetitive elements and their annotations into an easy-to-use web-browsable database system, RepPop [34] (Figure 2). RepPop is and will continue to be under continuing update on quarterly basis for annotation and curation of these repetitive elements. The composition of the RepPop database can be found in Table 1. We provided a Wiki interface for the whole community to help curate the annotations [35].

\section{Data browsing}

A user may browse all the 9,623 repetitive elements in the browsing interface of RepPop, as shown in Figure 3. The detailed annotation of each repetitive element can be retrieved using a popup window by clicking the corresponding entry under REName. Some repetitive elements have as high as 8,455 copies (RepPop694) in the Populus trichocarpa genome. We believe that it is not necessary to list the information of all the copies for such repetitive elements. So the browsing interface lists the information of at most 5 copies for each repetitive element as the default. The user can get the additional information, if needed, of all the copies by clicking the button "Get all". The user could also choose to browse only one of the following types of repetitive elements, namely, transposons, RNA genes, protein coding genes, and repetitive elements with 


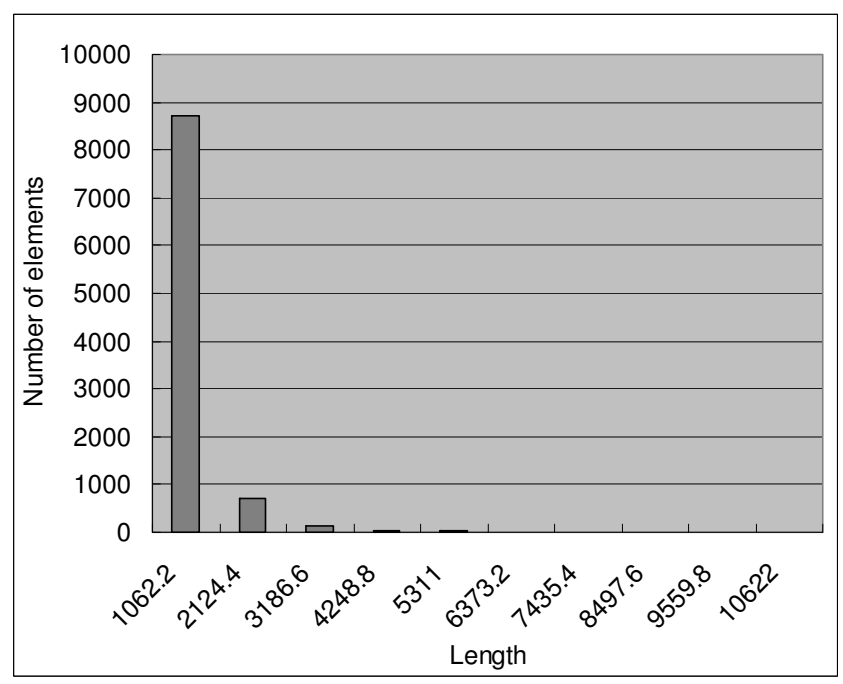

(a)

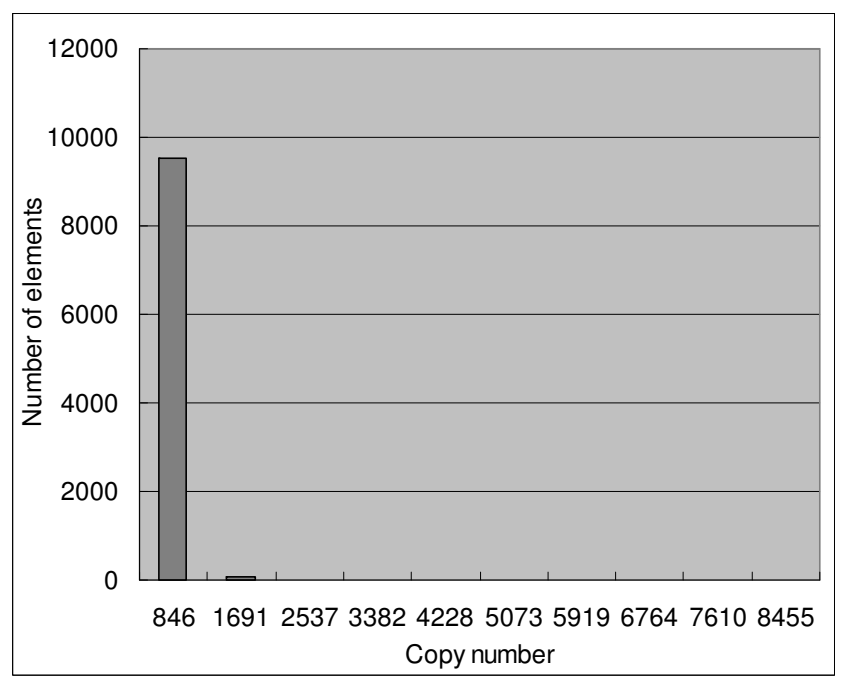

(c)

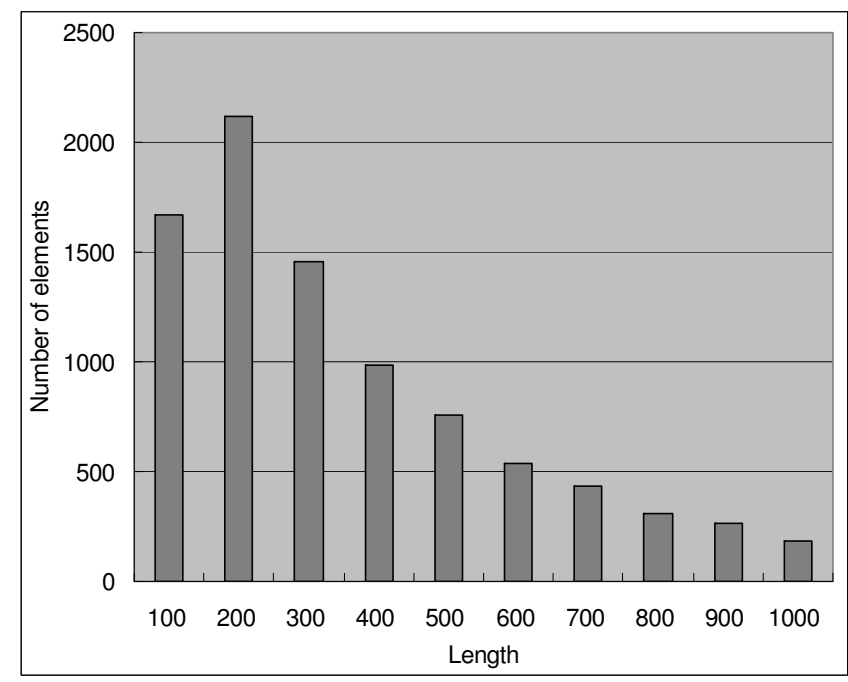

(b)

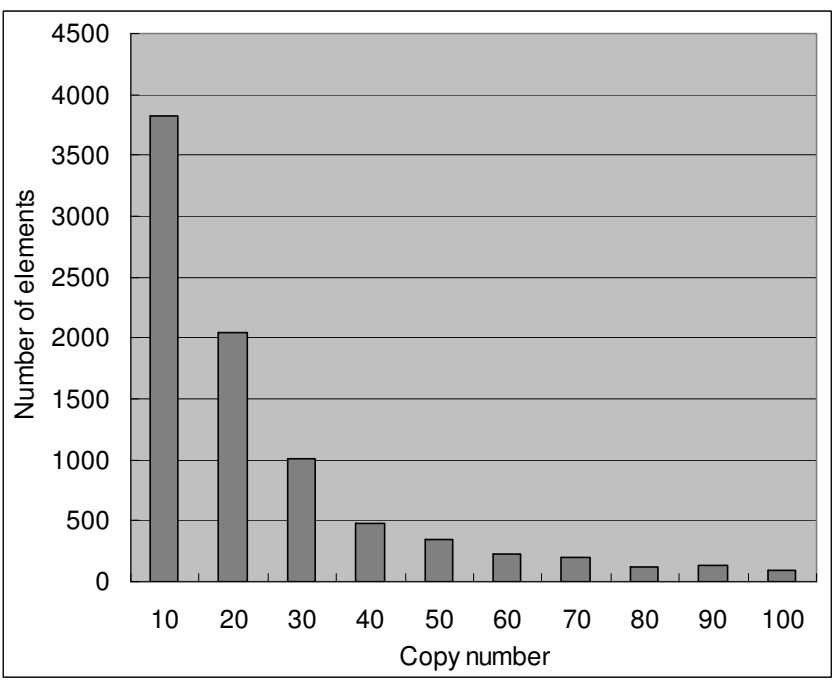

(d)

Figure I

Basic information of the identified $\mathbf{9 , 6 2 3}$ repetitive elements. Distributions of (a) lengths and (c) copy numbers of repetitive elements in the Populus trichocarpa genome. The two distributions within a shorter range are in (b) and (d).

Table I: Basic knowledge of the RepPop database

\begin{tabular}{|c|c|c|c|c|}
\hline Annotations & Number & Number\% & Length (bps) & Length\% \\
\hline Transposons & 161 & 1.67 & $21,044,639$ & 4.33 \\
\hline RNA genes & 15 & 0.16 & 36,051 & 0.01 \\
\hline Protein-coding genes & 2,983 & 31.00 & $157,586,923$ & 32.46 \\
\hline
\end{tabular}




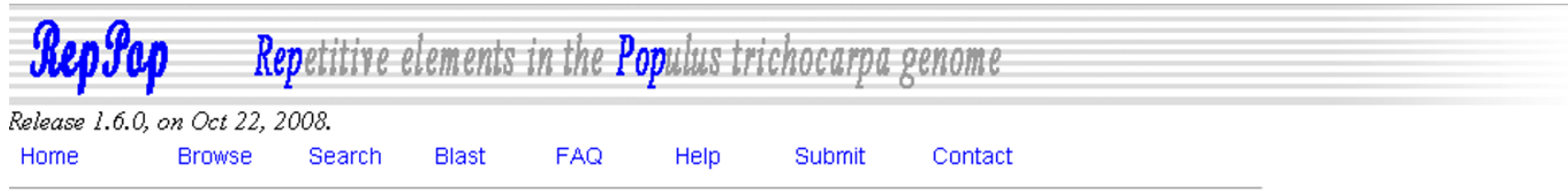

\begin{abstract}
The populus was selected as the first tree with the genome to be sequenced, mainly due to its small genome size, the wide deployment worldwide (30+ species), and its short juvenile period. Its rich content of cellulose, which is one of the most important source for biofuel. A female clone of $P$. trichocarpa was chosen to be sequenced.

The current assembly of Populus genome is release 1.0, whose small insert end-sequence coverage is $7.5 \mathrm{X}$, and it was released in June 2004 . It consists of 22,012 sequences (including the 19 chromosomes) and the total length is $485,510,911 \mathrm{bps}$. The data was downloaded from the offical site of the Populus trichocarpa genome sequencing project. The latest version of the genome can be found at the Poplar Genome Project at JGI Eukaryotic Genomics.

Duplication regions introduce significant difficulties into the correct assemblying of sequence contigs. For example, $\sim 45 \%$ of the $75.2-\mathrm{Mbp}$ segmental duplication regions in the initial assembly of the Norway Rat genome lacked chromosome assignment [1]. We identified all the repetitive elements in the populus genome. We further assign each of them as different classes of repetitive elements, including DNA transposons, RNA retrotransposons, Miniature Inverted-repeat Transposable Elements (MTE), Simple Sequence Repeats (SSR), and Segmental Duplications (SD), etc.

We organized the annotations into this easily browsable, searchable, and blastable database, Rep Pop, for the whole community. If you have any questions, please find the contact information in the Contact section.

We also provided a Wiki interface for the whole community to curate the annotations in this database at: http://csbl. bmb.uga.edu/ ffzhou/RepPopWiki/

References:

[1] Tuzun, E., et tal. Recent segmental duplications in the working draft assembly of the brown norway rat. Genome Research, 2004.
\end{abstract}

\title{
Figure 2
}

The main web page of database RepPop.

no annotations, by clicking the corresponding entry in the drop-down menu.

\section{Data search with key words}

The keyword search interface of RepPop makes it possible for a user to find items interesting to the user using a few keywords, as shown in Figure 4. Besides the typical keyword matching in the annotations, RepPop also provides the flexibility to support search for items within a specified range in terms of, say, elements with certain lengths or with certain copy numbers. For example, a pattern like "Length:Min-Max" as part of a keyword search can be used to find repetitive elements whose lengths are between the specified parameters Min and Max, whose default values are 0 and 10,622 if such range information is not specified. 10,622 is the maximum length of repetitive elements. Another example is including "Length $>100$ " as part of a keyword search to find repetitive elements with at least 100 bps; and similarly "Length $<500$ " is used to find elements with at most 500 bps. Similar range specifications are also available for specifying other quantities, say, "CopyNum:Min-Max" used for finding repetitive elements with copy numbers between specified parameters Min and Max. Tips for keyword searches are available in the Help box of RepPop.

\section{Sequence homology search}

An interface is provided to facilitate Blast search, using the NCBI Blast, against the 9,623 repetitive elements. Through this interface (Figure 5), a user can simultaneously specify up to 10 nucleotide or protein sequences of no more than $10 \mathrm{kbps}$ for homology search. A query example is provided for using this interface and can be found and used by clicking the button "Example (RepPop25)".

\section{User input to RepPop}

We were able to assign functions for 3,075 of the 9,623 repetitive elements, based on homology search against the NT database, leaving 6,548 ( 68.05\%) repetitive elements functionally unassigned. We have designed the RepPop interface in such a way that a user can submit his/her own functional annotations of any repetitive elements in RepPop through the Submission interface, as shown in Figure 6 . We have provided a Wiki web site for the general user community to directly annotate and curate the assigned functions of repetitive elements and to keep track of updates of annotations of each repetitive element, using PmWiki [36]. The developer of the RepPop database has the ultimate right to keep, revise or delete a particular contribution made by a user, which will be done on regular 


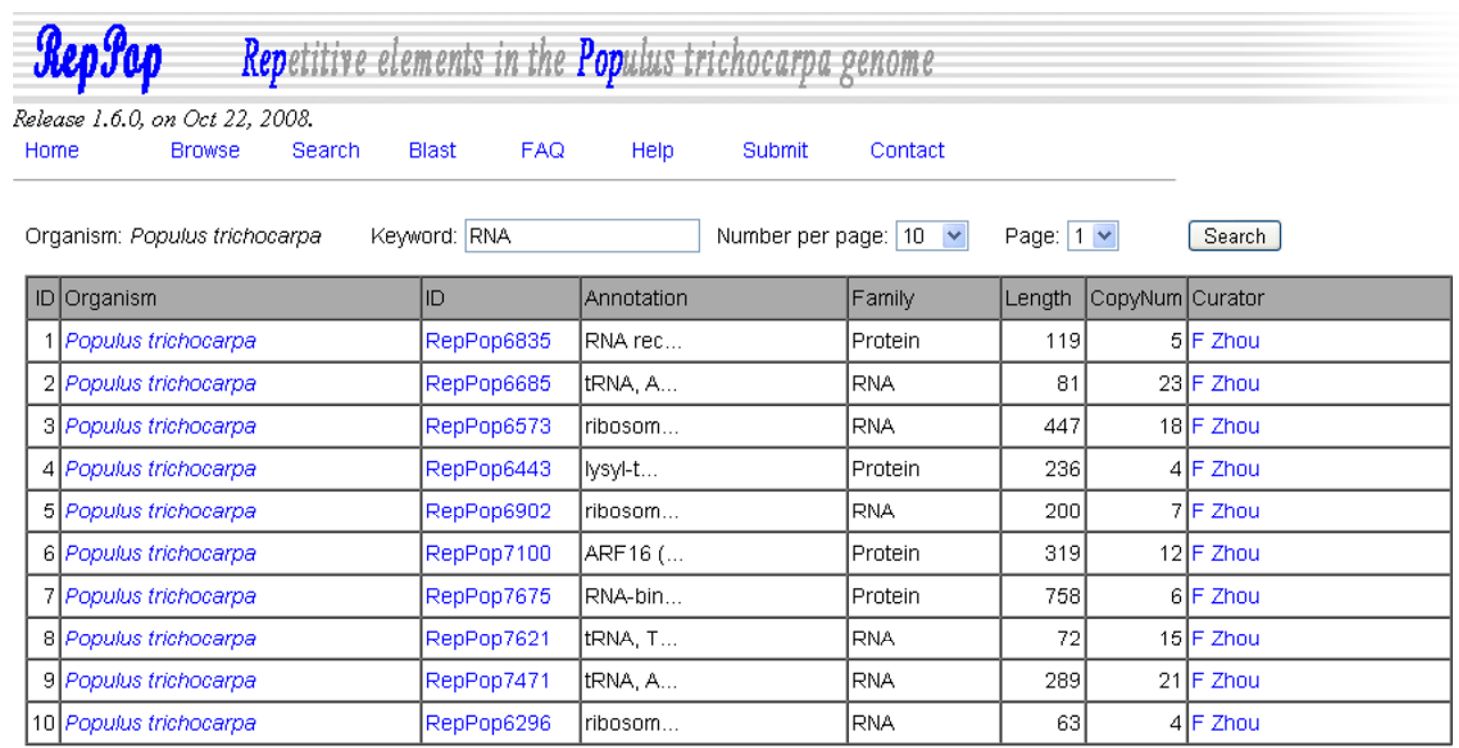

\section{Figure 4}

The searching interface of database RepPop. A user can search for repetitive elements with keywords through the keyword search interface.

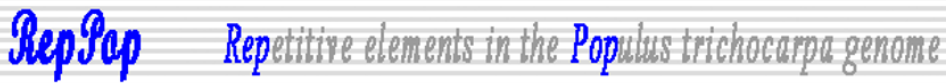

Release 2.6.0, on Oct 22, 2008.
Home Browse Search Blast FAQ Help Submit Contact

You could use this tool to verify whether your sequences are homologous to the repetitive elements in the Populus trichocarpa genome.

Please input your sequences here in FASTA format:

(Only the first 10 sequences with length $<=10,000 \mathrm{bps}$ will be processed)

$>$ RepPop25

TGATGCAACCATATTATTCGATAGTTTCACCCACATTTAACTAGTGTTTT

GCCTATGTTTTATATATABAATGCCTTGATATTCTTTGTTTTATGTTTTG

AAGGCACTTTTGGATGAAGGATGCABAAGGAGTAMATTGG AGATAATTG

GCAGATTTGACCTTCAG TCGATGTTTTG TGCAGAGCG TGAGCTCTAGAGG

TCGASATGAAGTGATTCCAGTGGCATTABASAGCTAACATCCATACCTTT

CTGGABATCTABCGCARGAAATAAATOAGGABGACCATGGABATCGCA

CIGGAABCTAG

C

CAACTIGARTACTGGAGCTACAGAAGTCCAMTTGATGCARCICAATTT

TTTTGGATTCCTGACTCAAAGACCTATAAACGCTCCAASTTCAGCCABA

AACGATGTCATATGAGGGAGATATGATTTTTCAAMGATGACAACTGASTT

CTGCCAGCAAACAGGTTTCG TGAAGARACGAG TCCAAATTACG TTCCGAS

GCATCTAAACCGACATCCAAGTTTTTATTTCAGCAATTTAGCTCCTCTAA

GTCABAGCTTGAAGATTTCATGCAAGGCTATTTCTCCTTTTTTAGGAAAS

TAGTTATTGAAGTACTTAAATGTAAACTG TCTACTTAAGGGAGGACTATT

\section{\begin{tabular}{lll}
\hline Reset & Example (RepPop25) \\
\hline
\end{tabular}}

Options:

$\begin{array}{ll}\text { Program: } & \text { blastn (nucleotide vs. nucleotide) } \checkmark \\ \text { E-value: } & 1 \mathrm{e}-5 \vee \\ \text { Filter: } & \square \text { Filter low complexity regions }\end{array}$

Reset Blast Example (RepPop25)

\section{Figure 5}

The blasting interface of database RepPop. Searches for the homologous regions for user-specified DNA or protein sequences in RepPop. 


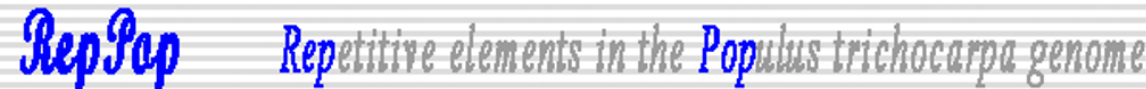

Release 1.6.0, on Oct 22, 2008.

Home Browse Search Blast FAQ Help Submit Contact

You could submit your comments about the annotations of the repetitive elements in this database RepPop

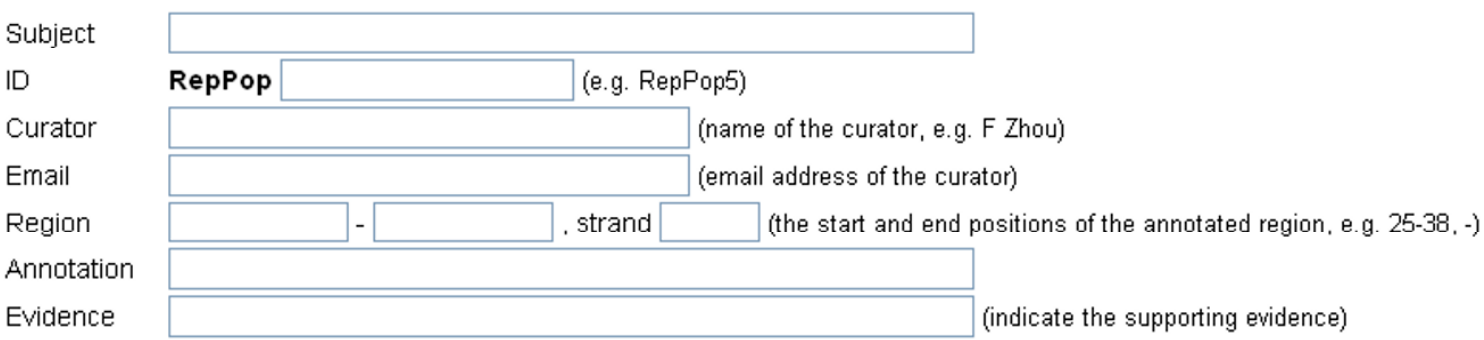

Submit

\section{Figure 6}

The submision interface of database RepPop. A user of RepPop may submit his/her annotations on a specific repetitive element with supporting evidence through this interface.

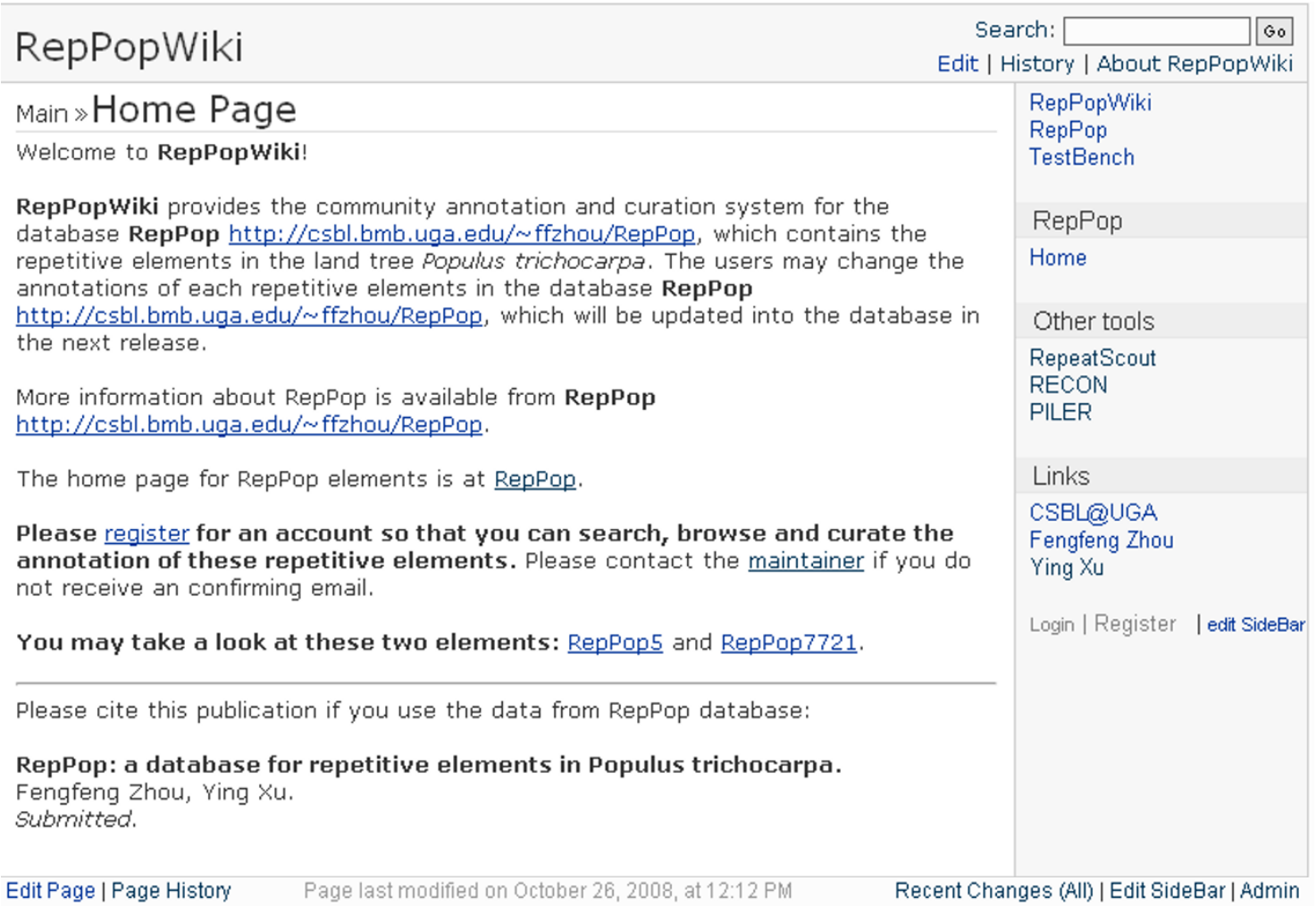

\section{Figure 7}

The Wiki interface of database RepPop. A user can revise the annotation of a specific repetitive element through this interface. 


\section{Future perspectives}

More efforts are being put into manual curations to provide more accurate annotations of the predicted repetitive elements, especially for the chimeric ones. Curations from other researchers, including users, are encouraged, as discussed above, through the web site of RepPop.

\section{Availability and requirement}

Project name: The repetitive elements in Populus trichocarpa genome.

Project home page: http://csbl.bmb.uga.edu/ ffzhou/ RepPop/.

Operating system(s): Platform independent.

Programming languages: PHP.

License: Not required.

Any restrictions to use by non-academics: None.

\section{Abbreviations}

RepPop: Repetitive elements in the Populus trichocarpa genome; IS: Insertion Sequence; LTR: Long Terminal Repeat.

\section{Authors' contributions}

FZ conceived the project, performed the identification and annotation of the data, and wrote the manuscript. YX wrote and polished the manuscript, and served as the principle investigator of the project. All authors have read and approved the final submitted version of this manuscript.

\section{Acknowledgements}

This work is supported in part by the National Science Foundation (DBI035477I, ITR-IIS-0407204, DBI-0542 I I9, CCF062 I 700), also National Institutes of Health (IROIGM07533I and IROIGM08I682) and a Distinguished Scholar grant from the Georgia Cancer Coalition, and the grant for the BioEnergy Science Center http://genomicsgtl.energy.gov/centers/ center ORNL.shtml, which is a U.S. Department of Energy Bioenergy Research Center supported by the Office of Biological and Environmental Research in the DOE Office of Science. We thank the colleagues in the Biofuel group of UGA CSBL for their comments on this work. We would also like to thank the two anonymous reviewers for helpful and constructive comments on our work.

\section{References}

I. Taylor G: Populus: arabidopsis for forestry. Do we need a model tree? Ann Bot (Lond) 2002, 90(6):68I-689.

2. Tuskan GA, Difazio S, Jansson S, Bohlmann J, Grigoriev I, Hellsten U, Putnam N, Ralph S, Rombauts S, Salamov A, et al.: The genome of black cottonwood, Populus trichocarpa (Torr. \& Gray). Science 2006, 3 I 3(5793): $1596-1604$.

3. Basso LC, de Amorim HV, de Oliveira AJ, Lopes ML: Yeast selection for fuel ethanol production in Brazil. FEMS yeast research 2008.

4. Li X, Weng JK, Chapple C: Improvement of biomass through lignin modification. Plant J 2008, 54(4):569-58I.
5. Schirmer-Michel AC, Flores SH, Hertz PF, Matos GS, Ayub MA: Production of ethanol from soybean hull hydrolysate by osmotolerant Candida guilliermondii NRRL Y-2075. Bioresource technology 2008, 99(8):2898-2904.

6. Smith DB, Flavell RB: Characterisation of the wheat genome by renaturation kinetics. Chromosoma 1975, 50(3):223-242.

7. Analysis of the genome sequence of the flowering plant Arabidopsis thaliana. Nature 2000, 408(68I4):796-8I5.

8. The map-based sequence of the rice genome. Nature 2005 , 436(7052):793-800.

9. Tuskan GA, Gunter LE, Yang ZK, Yin T, Sewell MM, DiFazio SP: Characterization of microsatellites revealed by genomic sequencing of Populus trichocarpa. Can J For Res 2004, 34(I):85-93.

10. Wicker T, Sabot F, Hua-Van A, Bennetzen JL, Capy P, Chalhoub B, Flavell $A$, Leroy $P$, Morgante $M$, Panaud $O$, et al: A unified classification system for eukaryotic transposable elements. Nature reviews 2007, 8( I 2):973-982

II. Ciampi MS, Schmid MB, Roth JR: Transposon TnI0 provides a promoter for transcription of adjacent sequences. Proc Natl Acad Sci USA 1982, 79( I 6):5016-5020.

12. Reynolds AE, Felton J, Wright $A$ : Insertion of DNA activates the cryptic bgl operon in E. coli KI2. Nature 198I, 293(5834):625-629.

13. Saedler H, Reif HJ, Hu S, Davidson N: IS2, a genetic element for turn-off and turn-on of gene activity in E. coli. Mol Gen Genet 1974, I 32(4):265-289

14. Louarn JM, Bouchâe JP, Legendre F, Louarn J, Patte J: Characterization and properties of very large inversions of the $E$. coli chromosome along the origin-to-terminus axis. Molecular \& general genetics: MGG 1985, 20 I (3):467-476.

15. Reif HJ, Saedler H: ISI is involved in deletion formation in the gal region of E. coli K12. Molecular \& general genetics: MGG 1975 , 137(I):17-28.

16. Schneider D, Duperchy E, Coursange E, Lenski RE, Blot M: Longterm experimental evolution in Escherichia coli. IX. Characterization of insertion sequence-mediated mutations and rearrangements. Genetics 2000, I56(2):477-488.

17. Li X, Heyer WD: Homologous recombination in DNA repair and DNA damage tolerance. Cell Res 2008, I 8(I):99-II3.

18. Smit A, Hubley R, Green P: RepeatMasker Open-3.0. 2004 [http:/ /www.repeatmasker.org].

19. Price AL, Jones NC, Pevzner PA: De novo identification of repeat families in large genomes. Bioinformatics 2005, 21 (Suppl I):i35I-358

20. Edgar RC, Myers EW: PILER: identification and classification of genomic repeats. Bioinformatics 2005, 2 I (SuppI I):i I 52-I 58

21. Levitsky VG: RECON: a program for prediction of nucleosome formation potential. Nucleic Acids Res 2004:W346-349.

22. JGI_Populus_trichocarpa_vI.I 2006 [http://genome.jgi-psf.org/ Poptrl I/Poptrl I.home.html].

23. Jurka J, Kapitonov VV, Pavlicek A, Klonowski P, Kohany O, Walichiewicz J: Repbase Update, a database of eukaryotic repetitive elements. Cytogenet Genome Res 2005, I I 0(I-4):462-467.

24. TREP 2008 [http://wheat.pw.usda.gov/ITMI/Repeats/].

25. Chaparro C, Guyot R, Zuccolo A, Piegu B, Panaud O: RetrOryza: a database of the rice LTR-retrotransposons. Nucleic Acids Res 2007:D66-70.

26. AtRepBase 1999 [http://nucleus.cshl.org/protarab/AtRep Base.htm].

27. Cole JR, Chai B, Farris RJ, Wang $\mathrm{O}$, Kulam-Syed-Mohideen AS, McGarrell DM, Bandela AM, Cardenas E, Garrity GM, Tiedje JM: The ribosomal database project (RDP-II): introducing myRDP space and quality controlled public data. Nucleic Acids Res 2007:D169-172

28. Griffiths-Jones S, Moxon S, Marshall M, Khanna A, Eddy SR, Bateman $A$ : Rfam: annotating non-coding RNAs in complete genomes. Nucleic Acids Res 2005:D I I - 124

29. Tanaka T, Antonio BA, Kikuchi S, Matsumoto T, Nagamura Y, Numa $\mathrm{H}$, Sakai $\mathrm{H}, \mathrm{Wu}$ J, Itoh T, Sasaki T, et al.: The Rice Annotation Project Database (RAP-DB): 2008 update. Nucleic Acids Res 2008:DI028-1033.

30. Wheeler DL, Barrett T, Benson DA, Bryant SH, Canese $K$, Chetvernin V, Church DM, Dicuccio M, Edgar R, Federhen S, et al.: Database resources of the National Center for Biotechnology Information. Nucleic Acids Res 2008:DI3-2I. 
31. Wootton JC, Federhen S: Analysis of compositionally biased regions in sequence databases. Methods Enzymol 1996, 266:554-57I.

32. Benson G: Tandem repeats finder: a program to analyze DNA sequences. Nucleic Acids Res 1999, 27(2):573-580.

33. Lowe TM, Eddy SR: tRNAscan-SE: a program for improved detection of transfer RNA genes in genomic sequence. Nucleic Acids Res 1997, 25(5):955-964.

34. RepPop 2008 [http://csbl.bmb.uga.edu/ ffzhou/RepPop/].

35. RepPopWiki 2008 [http://csbl.bmb.uga.edu/ ffzhou/RepPopWiki/].

36. PmWiki 2008 [http://www.pmwiki.org/].

Publish with Bio Med Central and every scientist can read your work free of charge

"BioMed Central will be the most significant development for disseminating the results of biomedical research in our lifetime. "

Sir Paul Nurse, Cancer Research UK

Your research papers will be:

- available free of charge to the entire biomedical community

- peer reviewed and published immediately upon acceptance

- cited in PubMed and archived on PubMed Central

- yours - you keep the copyright

Submit your manuscript here:

http://www.biomedcentral.com/info/publishing_adv.asp 